\title{
1 The ATLAS Fast Tracker and 2 Tracking at the High-Luminosity LHC
}

\author{
3 N. Ilic on behalf of the ATLAS collaboration \\ ${ }^{a}$ Stanford University \\ 450 Serra Mall, Stanford, CA 94305, USA \\ E-mail: Nikolina.Ilicecern.ch
}

The LHC's increase in centre-of-mass energy and luminosity in 2015 makes controlling trigger rates with high efficiency challenging. The ATLAS Fast TracKer (FTK) is a hardware processor built to reconstruct tracks at a rate of up to $100 \mathrm{kHz}$ and provide them to the high level trigger. The FTK reconstructs tracks by matching incoming detector hits with pre-defined track patterns stored in associative memory on custom ASICs. Inner detector hits are fitted to these track patterns using modern FPGAs. These proceedings describe the electronics system used for the FTK's massive parallelization. An overview of the installation, commissioning and running of the system is given. The ATLAS upgrades planned to enable tracking at the High-Luminosity LHC are also discussed.

KEYWORDS: LHC; ATLAS; Tracking. 
2.1 Operational Principle

2.3 Expected Performance 5

2.4 FTK Commissioning and Plans 5

3. Trigger Upgrades at High-Luminosity LHC 6

\section{Introduction}

From 2010 to 2012 the LHC operated at centre-of-mass energies of $7 \mathrm{TeV}$ and $8 \mathrm{TeV}$, colliding bunches of protons every 50 nanoseconds. ATLAS [1] is one of two multi-purpose detectors at the LHC built for a wide range of physics. Throughout its operation the ATLAS trigger system has efficiently selected interesting events, contributing to important results, including the discovery of the Higgs boson. From 2015 to 2018, the LHC will operate at $13 \mathrm{TeV}$ and $14 \mathrm{TeV}$, increase the luminosity by one order of magnitude, and collide bunches of particles every 25 nanoseconds, resulting in the mean number of simultaneous proton-proton interactions to double. This increase in luminosity and mean number of interactions during LHC operation is shown in Figure 1. Furthermore, during the 2021 to 2023 period of LHC operation the luminosity and number of simultaneous interactions will approximately double with respect to the previous run. In these environments, controlling the trigger rates and selecting interesting physics events with a high efficiency will be challenging. The ATLAS Fast TracKer (FTK), discussed in Section 2, is being installed and commissioned in order to cope with the challenge of selecting interesting physics events in the increasingly difficult environment. Another set of tracking and electronics upgrades are being developed in order to deal with 5-7 times higher luminosity that will be delivered at the High-Luminosity LHC in 2026. The ATLAS tracking at High-Luminosity LHC is discussed in Section 3.

\section{The ATLAS Fast Tracker}

The ATLAS trigger system consists of two levels: a hardware-based Level 1 (L1) trigger and a software-based High-Level trigger (HLT) [3]. At each level uninteresting events are rejected in order to efficiently reduce the amount of data in the final storage. The L1 trigger uses information from the muon tracks and electromagnetic and hadronic clusters to identify 

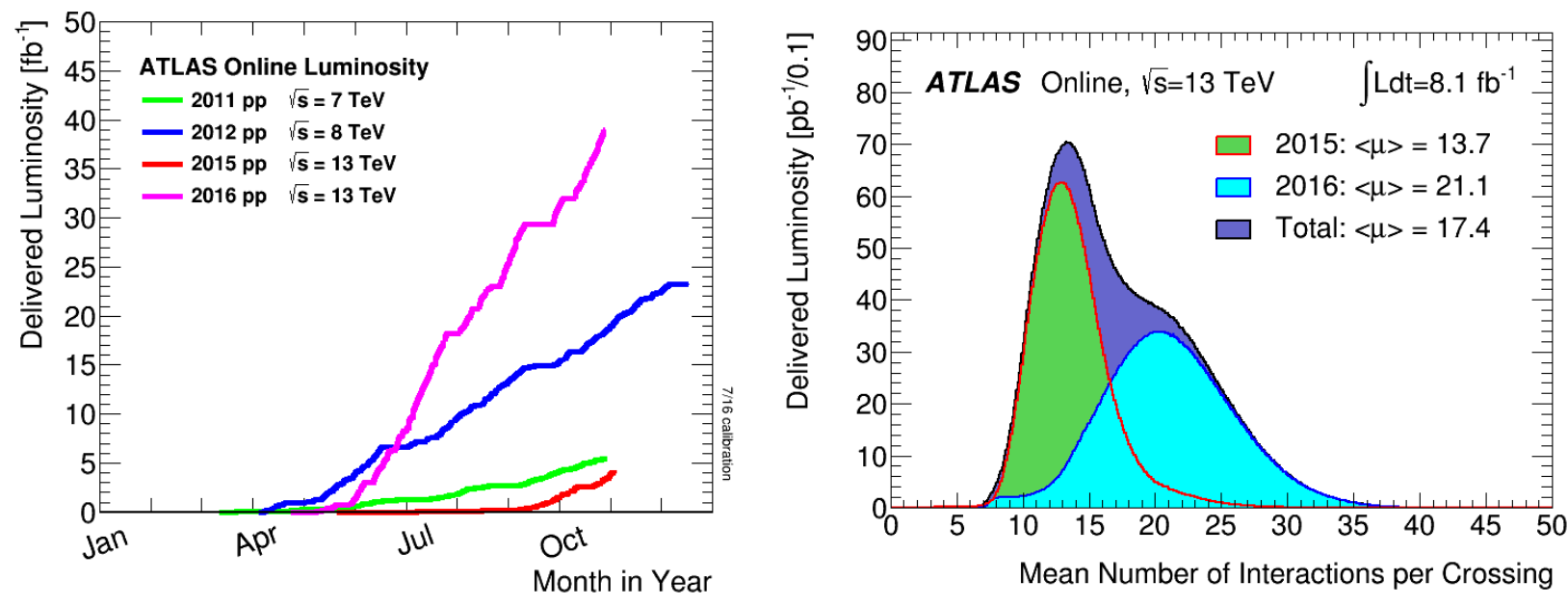

57

Figure 1: Left: The luminosity delivered by the LHC from 2011 to 2016 [2]. Right: The mean number of interactions in 2015 and 2016 [2].

interesting regions of the detector containing high energy deposits, called regions of interest. The HLT further rejects events by utilizing tracks from the inner detector in these interesting regions [4]. In the final step, the HLT decides which events to retain by reconstructing events using the energy deposits and tracks from the entire detector. The L1 trigger receives data from the ATLAS detector at a rate of $40 \mathrm{MHz}$, and outputs them to the HLT at a rate of $100 \mathrm{kHz}$, which further reduces it to $1 \mathrm{kHz}$ for permanent storage.

The Fast TracKer (FTK) is a new hardware system which will perform full track reconstruction after the L1 trigger, enabling the HLT to have access to tracks in the entire silicon detector [6]. By having access to all of the tracks earlier in the event selection stage, the trigger system can more efficiently reject uninteresting events. This greatly benefits physics analyses containing states identified using detailed tracking information, such as tau leptons and b-quarks.

There are many examples of physics analyses which will benefit from the tracking improvements the FTK will bring. It is important to measure Higgs properties and determine whether they match Standard Model (SM) expectations. For example, to uncover whether the fermionic couplings are SM-like, final states containing b-quarks and tau leptons are important but have large backgrounds from light quark and gluon jets. The FTK can help recognize bquarks by identifying displaced decay vertices which are characteristic of B-hadron decays, depicted on the left of Figure 2. The right of Figure 2 illustrates that hadronic tau decays contain fewer tracks in more narrow energy cones (jets) than other hadronic energy deposits. The FTK's ability to count tracks inside of cones of energy deposits leads to an improvement in tau identification. The FTK's improved b-quark and tau identification will also help SUSY analyses in which sparticles decay to medium energy b-quarks and tau leptons. In addition, the FTK will allow for the possibility of a track-based Missing Transverse Energy trigger, which is less sensitive to overlapping interactions and can benefit exotic and other analyses [7]. 






"typical" jet

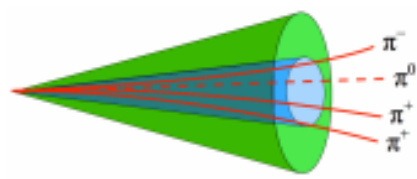

$\tau$ jet: fewer tracks in smaller cone

Figure 2: Left: A secondary vertex resulting from the decay of a B-hadron. Right: The many tracks in a larger cone shown for a typical jet versus the fewer tracks in a smaller cone characteristic of tau jets.

\subsection{Operational Principle}

The FTK receives hits from the twelve layers of the ATLAS Pixel detector and Semiconductor Tracker (SCT) and clusters them in order to reduce the data size. The clustered hits are then sorted into 64 regions and sent downstream for parallel processing. For part of the processing, the Pixel and SCT detectors are not viewed as individual modules, but groupings of modules called super strips. For eight of the twelve layers, the processing units identify which super strips the clustered hits belong to. The selected super strips are then compared to MC track patterns stored in associated memories. The MC track patterns that match the selected super strips are called roads. For each road the fine resolution hits are retrieved and a goodness of fit is determined using a $\chi^{2}$ test for eight layers. The eight-layer tracks are then combined with the hits from the remaining four layers and a full twelve-layer track fit is performed. The twelve-layer tracks are then sent to the HLT. Figure 3 shows a visual representation of the process.

\subsection{Hardware Components}

The Input Mezzanines (IM) are 128 boards that receive the inner detector data at the 100 $\mathrm{kHz}$ input event rate and cluster them. Using a combination of Spartan-7 and Artix-7 FPGAs, the IMs calculate the cluster centroid, shape and size and information that contain the charge of the particle. The IM boards sit on the Data Formatter (DF) boards which sort the data into 64 regions using Xilinx Virtex-7 FPGAs. The final system will contain 32 DF boards in ATCA shelves, distributing data to one another using the ATCA full-mesh backplane and inter-shelf fibres. A custom microcontroller is used for board powering, sensor control and remote FPGA programming. The top left of Figure 4 shows the DF board with four IMs on it. 


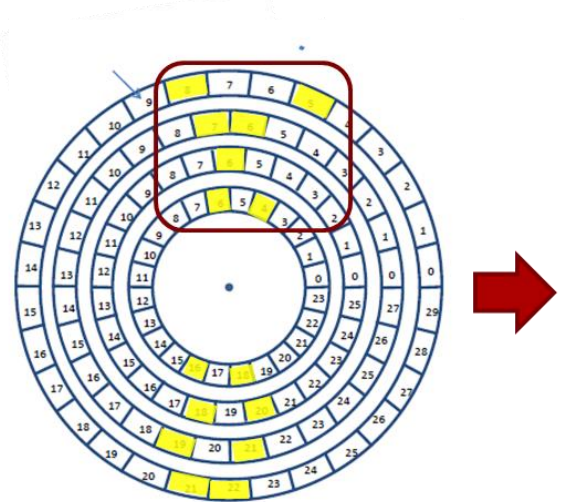

Identify hits by coarser grained Pix/SCT segments called super strips (SS)



Find pre-stored MC track patterns that match the super strips in 8 layers. Matches are called Roads
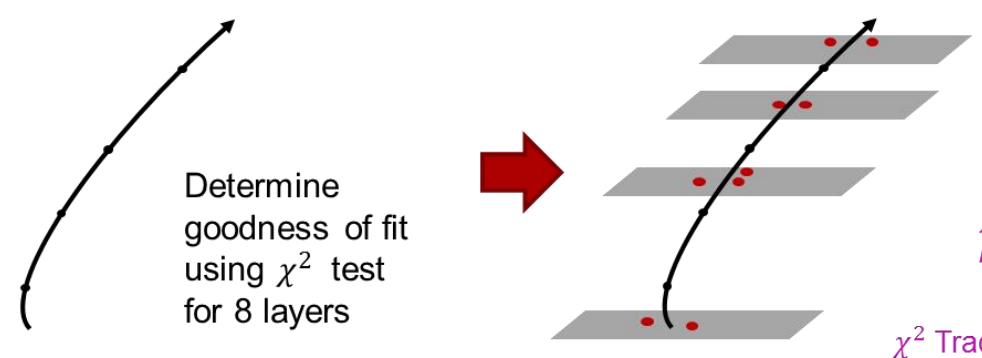

Extrapolate 8 layer tracks to additional 4. Perform 12 layer track fit

$$
p_{i}=\sum_{j} C_{i j} \cdot x_{j}+q_{i}
$$

Figure 3: A summary of the FTK's operational principle.

The Associative Memory performs the super strip to MC track pattern matching for eight inner detector silicon layers. Each Associative Memory board (AMB) contains four Local Associative Memory boards which each hold 16 Associative Memory chips. The chips store 1 billion patterns in HEP-specific Content Addressable Memory (CAM), and perform $10^{14}$ word comparisons per second. The chips also allow for patterns with widths that vary layer by layer using ternary CAM cells. The final system will consist of 128 AMB boards stored in VME crates. An AMB board with its four LAMBs is shown in the top middle of Figure 4.

The Auxilary (AUX) board receives roads from the AMB, retrieves the full resolution hits and does track fitting in eight layers. The AUX board rejects tracks below a certain $\chi^{2}$ in order to reduce data to the next board. It also accounts for tracks that do not have hits in every layer allowing them to be dropped. The AUX uses Aletera Arria-5 FPGAs and will consist of 128 boards in VME crates. An AUX card is shown in the top right of Figure 4.

The second stage board receives eight-layer tracks from the AUX and hits from the remaining four layers from the DF. It uses Xilinx Kintex-7 FPGAs to extrapolate the eight layer tracks to the additional layers and does the full twelve-layer track fitting. The final system will consist of 32 SSB boards, one of which is shown in the bottom right of Figure 4.

The FTK Level-2 Interface (FLIC) organizes tracks from the SSB and sends them to the High-Level Trigger Readout systems. It uses two custom microcontrollers and Xilinx Virtex-6 FPGAs. The final system will have 2 FLIC boards in ATCA shelves, one of which is shown in the bottom middle of Figure 4. 

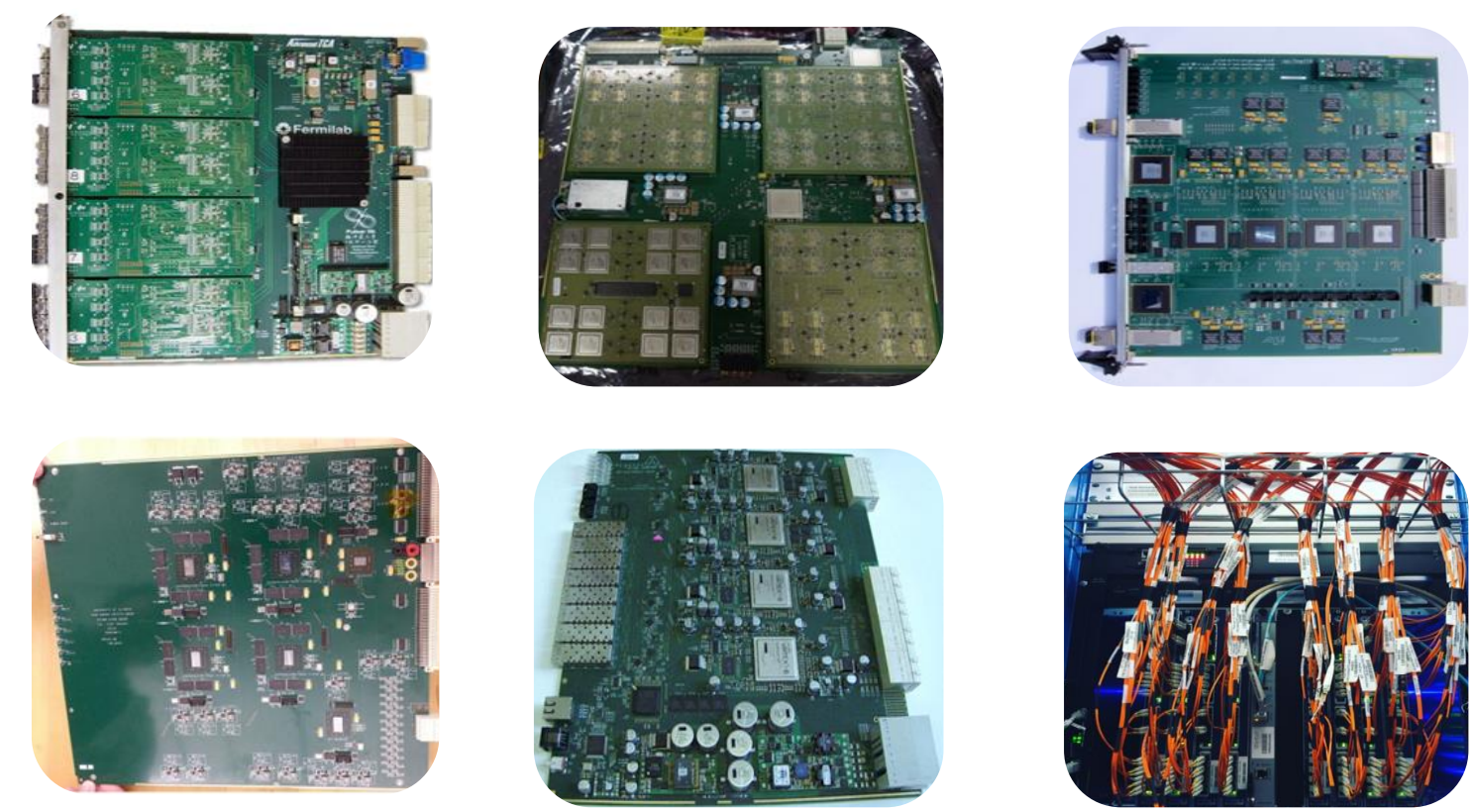

Figure 4: Top Left: DF with four IMs on it. Middle Left: AMB with 4 LAMBs on it and 16 chips per LAMB. Top Right: AUX Card. Bottom Left: SSB. Bottom Right: Fully installed ATCA shelf.

\subsection{Expected Performance}

The FTK reconstruction efficiency with respect to offline reconstruction is greater than $90 \%$ as can be seen from Figures 5. The left of Figure 5 shows that the offline and FTK variance of the $d_{0}$ parameter as a function of the particle charge over its momentum closely match. The right of Figure 5 shows that the efficiency with respect to offline for a muon and pion as a function of their momentum is greater than $90 \%$. The small differences are due to using only silicon detectors, using a simplified clustering model and considering only tracks with momentum greater than $1 \mathrm{GeV}$.

\subsection{FTK Commissioning and Plans}

The FTK is currently being installed and commissioned in the electronics room beside the ATLAS caverns. Currently $65 \%$ of the IM+DF boards are installed in their ATCA shelves. An example of a full DF ATCA shelf is shown in the bottom right of Figure 4. A slice containing IMs, a DF and AUX board is currently integrated with the ATLAS detector and regularly taking data. The hits from this slice are written into the ATLAS event data stream. The firmware for all of the systems is complete and undergoing debugging with simulated data as well as real LHC data. The communication between the different boards is being stress tested in a separate laboratory that mimics the setup of the electronics room beside the cavern. The data flowing through all of the boards are being compared to the data expected from simulation. By the end of 2016 a part of the FTK system will be stably taking data with the ATLAS detector. In 2017 the full detector is expected to be commissioned using half of the processing units (64 AUX+AMB pairs), and the ATLAS trigger is planned to use the FTK. By 2018 the full detector is planned to be commissioned with all of the processing units. 



Figure 5: Left: Comparison of offline to FTK reconstruction for the variance of $\mathrm{d}_{0}$ vs charge over momentum [5]. Right: FTK efficiency with respect to offline for a muon and a pion versus its momentum [5].

\section{Trigger Upgrades at High-Luminosity LHC}

In 2026 the LHC will resume operation after it has been upgraded to operate at 5 to 7 times the nominal 2016 luminosity. The number of interactions per bunch crossing at the HighLuminosity LHC is expected to rise to 200. The current single lepton triggers are not able to deal with this high rate at their current momentum thresholds. Simply increasing the momentum threshold below which events are rejected would lead to large loss in physics events. Thus several upgrades, including tracking information at the hardware based L1 trigger are being developed in order to help ATLAS deal with the increase in luminosity.

A new all-silicon tracker, called the ATLAS Inner Tracker (ITK) will replace the inner detector. In the calorimeter front-end electronics will be replaced in order to enable sending finer granularity information to the trigger at a $40 \mathrm{MHz}$ rate. The muon spectrometers will also be upgraded to include new resistive plate chambers to allow for a larger acceptance, and the use of muon drift tubes in the first level of the muon trigger. Finally, FTK ++ is an upgraded version of the FTK with newer FPGAs and a larger number of patterns. It will act as an HLT co-processor, providing global track reconstruction for tracks above $1 \mathrm{GeV}$, when requested by the HLT.

The upgrade for the High-Luminosity LHC is currently being shaped and various schemes are being studied [8]. Figure 6 shows a schematic of a possible new system. An L0 trigger would be introduced and operate at $1 \mathrm{MHz}$ with a $6 \mu$ s latency. The $\mathrm{L} 1$ trigger would reduce the rate to $400 \mathrm{kHz}$ with a latency of $24 \mu \mathrm{s}$. The L1 trigger would consist of an L1 track trigger, complementary to FTK++, and will perform tracking in regions of interest for tracks with momentum above $4 \mathrm{GeV}$. The L1 track would feed the L1 Global trigger which would process finer granularity calorimeter information to improve the electron/photon/tau/jets measurement. 


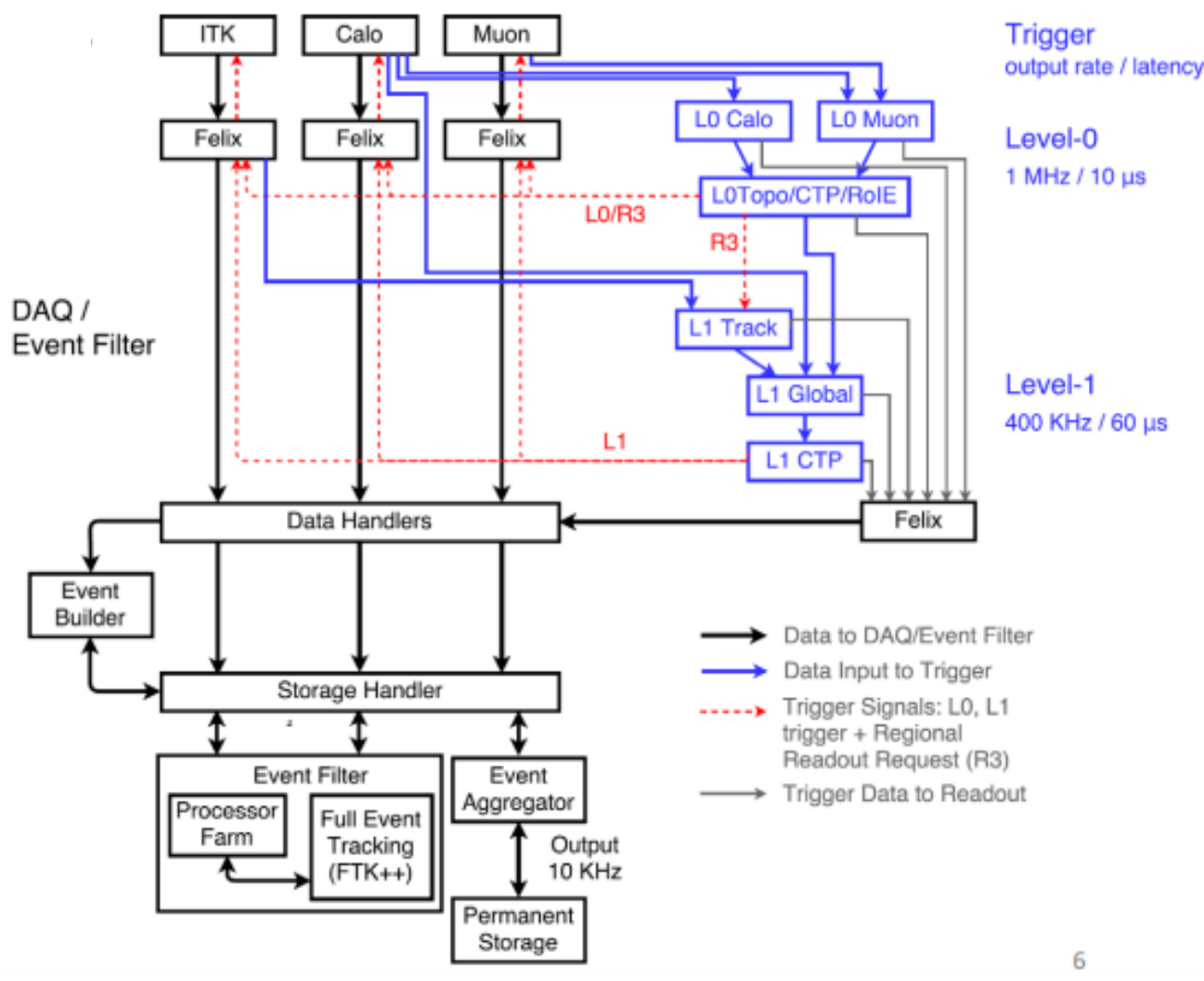

Figure 6: Possible changes that could be implemented in the trigger system for the High-Luminosity LHC upgrade.

\section{References}

[1] ATLAS Collaboration, The ATLAS Experiment at the CERN Large Hadron Collider, JINST 3 (2008) S08003

[2] https://twiki.cern.ch/twiki/bin/view/AtlasPublic/LuminosityPublicResultsRun2

[3] ATLAS Collaboration, ATLAS detector and physics performance: Technical Design Report, CERN-LHCC-99-014

[4] ATLAS Collaboration, ATLAS Inner Detector : Technical Design Report, CERN-LHCC-97-16

[5] https://twiki.cern.ch/twiki/bin/view/AtlasPublic/FTKPublicResults

[6] ATLAS Collaboration, Fast TracKer (FTK) Technical Design Report, CERN-LHCC-2013-007

[7] ATLAS Collaboration, Measurement of the missing transverse momentum based on tracks in proton-proton collisions at $\operatorname{sqrt}(\mathrm{s})=900 \mathrm{GeV}$ centre-of-mass energy with the ATLAS detector, ATLAS-CONF-2010-020

[8] ATLAS Collaboration, ATLAS Phase-II Upgrade Scopincg Document, CERN-LHCC-2015-020 